\title{
Superhydrophobic Carbon Nanotube Forests
}

\author{
Kenneth K. S. Lau, ${ }^{* \dagger}$ José Bico, ${ }^{\ddagger}$ Kenneth B. K. Teo,§ Manish Chhowalla," \\ Gehan A. J. Amaratunga, ${ }^{\S}$ William I. Milne,$\S$ Gareth H. McKinley, ${ }^{\ddagger}$ and \\ Karen K. Gleason ${ }^{\dagger}$ \\ Department of Chemical Engineering, Massachusetts Institute of Technology, \\ Cambridge, Massachusetts 02139, Department of Mechanical Engineering, \\ Massachusetts Institute of Technology, Cambridge, Massachusetts 02139, Engineering \\ Department, University of Cambridge, Cambridge CB2 1PZ, United Kingdom, and \\ Department of Ceramics and Materials Engineering, Rutgers University, \\ Piscataway, New Jersey 08854
}

Received August 26, 2003; Revised Manuscript Received October 8, 2003

\begin{abstract}
The present study demonstrates the creation of a stable, superhydrophobic surface using the nanoscale roughness inherent in a vertically aligned carbon nanotube forest together with a thin, conformal hydrophobic poly(tetrafluoroethylene) (PTFE) coating on the surface of the nanotubes. Superhydrophobicity is achieved down to the microscopic level where essentially spherical, micrometer-sized water droplets can be suspended on top of the nanotube forest.
\end{abstract}

Since their discovery in $1991,{ }^{1}$ carbon nanotubes continue to be a subject of unabated scientific research and development. Their extraordinary properties ${ }^{2-4}$ make them highly attractive as technology enablers in a host of different applications, ranging from fillers in polymer nanocomposites to conductors in molecular electronics. ${ }^{5}$ Carbon nanotubes can be single-walled (SWNTs) or multiwalled (MWNTs), metallic or semiconducting, and isolated or attached to a substrate. The ability to grow nanotubes directly on a substrate using various chemical vapor deposition techniques allows the production of high purity nanotubes in a controlled manner. Added to this, the ability to functionalize the surface of individual nanotubes can create synergistic effects in nanotube properties. Here we report the creation of a superhydrophobic surface via functionalization of vertically aligned carbon nanotubes with a nonwetting poly(tetrafluoroethylene) (PTFE) coating. Vapor condensation experiments inside an environmental scanning electron microscope (ESEM) confirmed that the superhydrophobic effect is observable even for microscopic water droplets. We further demonstrate that this superhydrophobicity on a functionalized forest can be achieved with relatively short nanotube heights.

Such superhydrophobicity can be understood by observing nature. In certain plant leaves, such as the lotus leaf (Nelumbo nucifera), rain droplets are known to roll or bounce off these

\footnotetext{
* Corresponding author. E-mail: klau@mit.edu.

Department of Chemical Engineering, MIT.

$\doteqdot$ Department of Mechanical Engineering, MIT.

$\S$ University of Cambridge.

"Rutgers University.
}

leaves, removing dust particles and surface contaminants. This self-cleaning or Lotus effect ${ }^{6,7}$ is caused by both the hierarchical roughness of the leaf surface from micrometersized papillae having nanometer-sized branch like protrusions and the intrinsic material hydrophobicity of a surface layer of epicuticular wax covering these papillae. ${ }^{8}$ A very rough, heterogeneous surface allows air to be trapped more easily underneath the water droplet so the droplet essentially rests on a layer of air. A significantly higher surface area compared to the projected area in the case of a rough surface requires a greater energy barrier to create a liquid-solid interface. Coupled to this, when the surface energy of the surface material is intrinsically low, the combined effect is that the surface will repel any water that comes into contact with it. ${ }^{9,10}$

Likewise, our PTFE-coated carbon nanotube forests aim to mimic nature's design. By growing a forest of nanotube pillars, an organized, heterogeneous surface is synthesized on a nanoscale. This makes it easy even for extremely small water droplets to be suspended on a surface approaching that of a perfect air-water interface (contact angle of $\left.180^{\circ}\right) .{ }^{11}$ By coating this forest template with a PTFE layer, the water comes into contact with a material having one of the lowest surface energies $(18 \mathrm{mN} / \mathrm{m})$ and thus a high contact angle ( $108^{\circ}$ on a smooth PTFE surface). ${ }^{12}$ By combining these two elements, the PTFE-coated nanotube forest can prevent water penetration down to the microscopic level, creating an enhanced superhydrophobic effect. Such modified carbon nanotubes may potentially be used in microfluidic devices, 

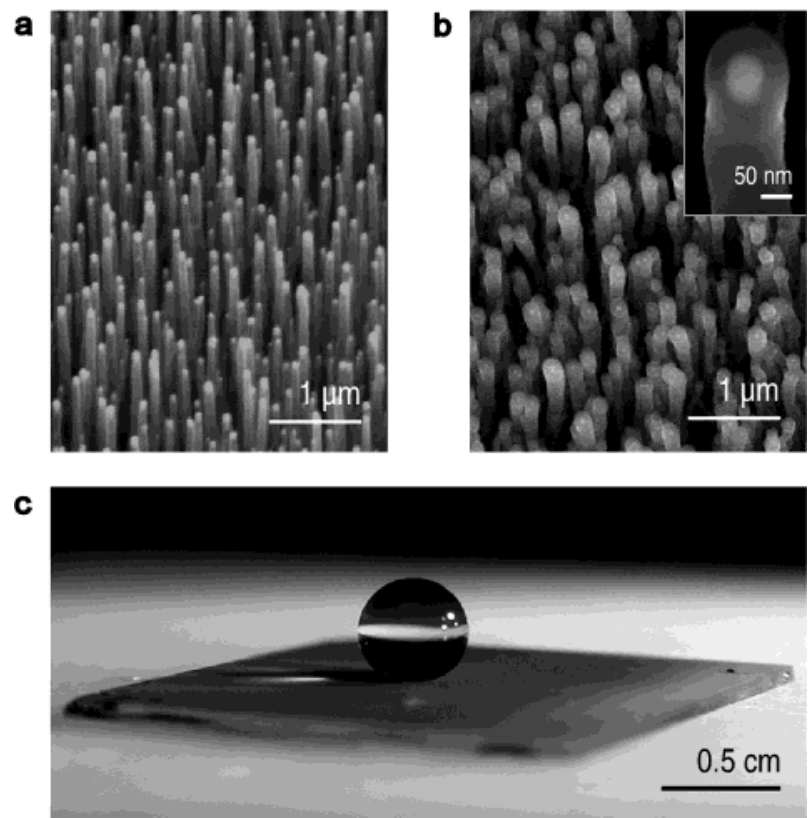

Figure 1. SEM images of carbon nanotube forests. (a) As-grown forest prepared by PECVD with nanotube diameter of $50 \mathrm{~nm}$ and a height of $2 \mu \mathrm{m}$, (b) PTFE-coated forest after HFCVD treatment, and (c) an essentially spherical water droplet suspended on the PTFE-coated forest.

antisoiling or antifouling surfaces, efficient heat transfer areas, or nonbinding biopassive surfaces.

We deposited the vertically aligned carbon nanotube forest with a plasma enhanced chemical vapor deposition (PECVD) technique. ${ }^{13-15}$ Although a variety of different methods are also currently available, the PECVD process is the only technique that produces perfectly aligned, untangled (i.e., individually standing) carbon nanotubes whose height and diameter can be conveniently controlled. The PECVD process can be summarized in two main steps. First, the formation of nickel $(\mathrm{Ni})$ catalyst islands on an oxidized (20 $\mathrm{nm})$ silicon substrate through the sintering of a thin $(5 \mathrm{~nm})$ $\mathrm{Ni}$ film at $650{ }^{\circ} \mathrm{C}$. Second, nanotube growth from these discrete catalyst islands in a DC plasma discharge (bias -600 $\mathrm{V})$ of acetylene and ammonia, using flow rates of 75 and $200 \mathrm{sccm}$, respectively, at a partial pressure of 4 Torr. The PECVD process enables the growth of nanotubes aligned in the vertical direction. The nanotube diameter and areal density are controlled by the initial thickness of the $\mathrm{Ni}$ catalyst layer, with a thinner film leading to narrower nanotubes of higher density. The nanotube height is controlled by the plasma deposition time (typical nanotube growth rate is $330 \mathrm{~nm} / \mathrm{min}$ ). Figure 1a illustrates a typical nanotube forest grown through this process as viewed under scanning electron microscopy (SEM) using a Hitachi S800FE SEM operating at $20 \mathrm{kV}$. The sample has an areal density of 10 MWNTs per $\mu \mathrm{m}^{2}$, with the vertical MWNTs having a mean diameter of $50 \mathrm{~nm}$ (as-grown) and a height of $2 \mu \mathrm{m}$.

An array of such relatively short nanotubes is not sufficiently hydrophobic on its own; on the contrary, water droplets deposited on the surface immediately penetrate into the forest. This is presumably due to the high surface energy of the nanotubes, essentially a graphite material (contact angle of $84-86^{016,17}$ ), that causes the water to seep into the voids of the forest. Further, microscopic examination of such samples after drying reveals that the nanotubes are forced into bundles under the surface tension effects of the evaporating water between the nanotubes, confirming our hypothesis. Our observation may appear contradictory with experiments on tall carbon nanotubes grown off a substrate where superhydrophobicity has been observed. ${ }^{18,19}$ Thus, we investigated taller $(10-15 \mu \mathrm{m})$ nanotube forests, and these surfaces in the as-grown state did give an initial water contact angle of $161^{\circ}$. However, the droplets are not stable and eventually seep into the forest voids after a few minutes. The apparent superhydrophobicity of the taller nanotubes most likely results from secondary roughness, as there is a larger variation in height of the taller nanotubes which was observed through electron microscopy. The eventual penetration of the water droplets is due to the high surface energy of the nanotube's graphitic surface. This suggests that PTFE functionalization is a necessary step for making a stable superhydrophobic surface.

The PTFE coating is applied onto the forest of carbon nanotubes through a hot filament chemical vapor deposition (HFCVD) process. ${ }^{20,21}$ The process coats along the height of carbon nanotubes with a sufficiently thin PTFE coating, unlike conventional methods in which greater minimum coating thicknesses $(>10 \mu \mathrm{m})$ can smooth out the surface texture. Using an array of stainless steel filaments resistively heated to $500{ }^{\circ} \mathrm{C}$, hexafluoropropylene oxide (HFPO) gas is thermally decomposed to form difluorocarbene $\left(\mathrm{CF}_{2}\right)$ radicals. These radicals polymerize into PTFE on the nanotube forest substrate that is kept at room temperature. An initiator, perfluorobutane-1-sulfonyl fluoride, is used to promote the polymerization process. Flow rates of HFPO and the initiator are maintained at 23 and $6 \mathrm{sccm}$, respectively, and pressure is kept at 0.5 Torr. Figure 1b shows an SEM micrograph of the $2 \mu \mathrm{m}$ tall nanotube forest after being coated with PTFE. Each individual pillar is seen to be coated uniformly and the forest structure is preserved. Unlike the as-grown forests, the treated forests show stable superhydrophobicity, yielding nearly spherical water droplets on a macroscopic level when water is deposited on the surface, as shown in Figure 1c. The advancing and receding contact angles of the treated forest shown here are $170^{\circ}$ and $160^{\circ}$, respectively. Contact angle measurements are performed using the sessile drop method; the water droplets are introduced using a microsyringe and images are captured to measure the angle of the liquid-solid interface.

Fourier transform infrared spectroscopy (FTIR) confirms that our PTFE coating is essentially identical to bulk PTFE, see Figure 2. FTIR spectra are acquired using a Thermo Nicolet NEXUS 870 equipped with a DTGS detector at $4 \mathrm{~cm}^{-1}$ resolution. The spectrum of the as-grown carbon nanotube forest shows no distinctive FTIR peaks. The spectrum of the PTFE-coated forest in contrast shows strong absorptions of the symmetric and asymmetric $\mathrm{CF}_{2}$ stretches in the $1250-1150 \mathrm{~cm}^{-1}$ region characteristic of bulk PTFE. ${ }^{22,23}$ Other methods, such as PECVD or laser ablation, may yield thin fluorocarbon coatings that can also cover the 


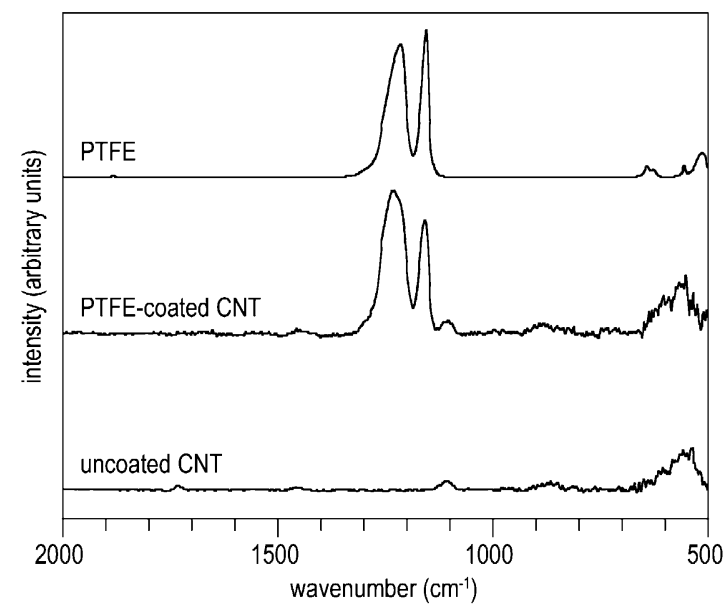

Figure 2. FTIR spectra of carbon nanotube forests. The spectrum of the PTFE-coated forest shows $\mathrm{CF}_{2}$ related peaks similar to the spectrum of bulk PTFE. The spectrum of the as-grown forest shows no distinctive peaks.

entire length of the nanotubes, but these coatings, unlike HFCVD, suffer from poor compositional resemblance to bulk PTFE.

Aside from static experiments, we were interested in the dynamic behavior of water on the PTFE-treated carbon nanotube forest. On a macroscopic level, we compared the behavior of a water droplet free falling on the untreated and the PTFE-treated forest. This is captured using a PhotoSonics Phantom V5.0 high-speed camera at a $1000 \mathrm{~Hz}$ frame rate and selected time sequence of images are presented in Figure 3 (video clips can be viewed from ref 24). Figure 3a shows the behavior of a droplet falling on the as-grown nanotube forest during its first impact. The droplet advances with a contact angle greater than $90^{\circ}$ (panels 2 and 3) but recedes with an angle less than $90^{\circ}$ (panels 4 and 5), and eventually comes to rest by wetting the surface (panel 6). Figure $3 \mathrm{~b}$ reveals a significantly different behavior on a PTFE-coated nanotube forest, showing the droplet advances (panels 2 and 3) and recedes (panel 4) with an angle greater than $90^{\circ}$. The surface is so superhydrophobic that the droplet, on receding, actually has sufficient momentum to leave the surface (panel 5). After several more bounces (not shown), the droplet eventually bounces off without ever coming to rest on the surface (panel 6). ${ }^{25,26}$

On a microscopic level, we used ESEM to observe the behavior of water condensing on our nanotube forest surface. By precisely controlling the water vapor pressure (5.5 Torr) and the temperature of the sample stage $\left(3.4{ }^{\circ} \mathrm{C}\right)$ in the Philips/FEI XL30 FEG ESEM chamber, we were able to image the formation of micrometer-sized water droplets at $30 \mathrm{kV}$, as seen in Figures $4 \mathrm{a}$ and $4 \mathrm{~b}$ from a top-down and $15^{\circ}$ tilt views, respectively. Figures $4 \mathrm{c}$ and $4 \mathrm{~d}$ capture remarkably close-up views, both from top-down and at a $15^{\circ}$ tilt, of water droplets $(3-4 \mu \mathrm{m}$ in diameter) that are essentially spherical and clearly suspended on top of the template of carbon nanotubes which are also visible, demonstrating that we have created a carbon nanotube forest that is superhydrophobic down to the micrometer dimension (video clip can be viewed from ref 24). Repeated cycles of condensation and evaporation did not have any effect on the structure of the PTFE-treated forests, and spherical water droplets can be condensed and evaporated reversibly. In contrast, as mentioned earlier, upon water or a wetting volatile liquid exposure, untreated forests are typically found to bundle together under surface tension forces during drying, see Figure 5. Nanotubes bend to form clusters at the top while the roots remain attached to the underlying surface, and if the surface tension forces are strong enough, the nanotubes snap off. This further highlights the need for the PTFE treatment to ensure that liquid surface tension effects do not irreversibly and detrimentally alter the forest template structure.

Quantitatively, model surfaces with a controlled topography suggest a very simple relation (Cassie-Baxter equation $^{27}$ ) between the apparent contact angle $\theta^{*}$ observed on a

a
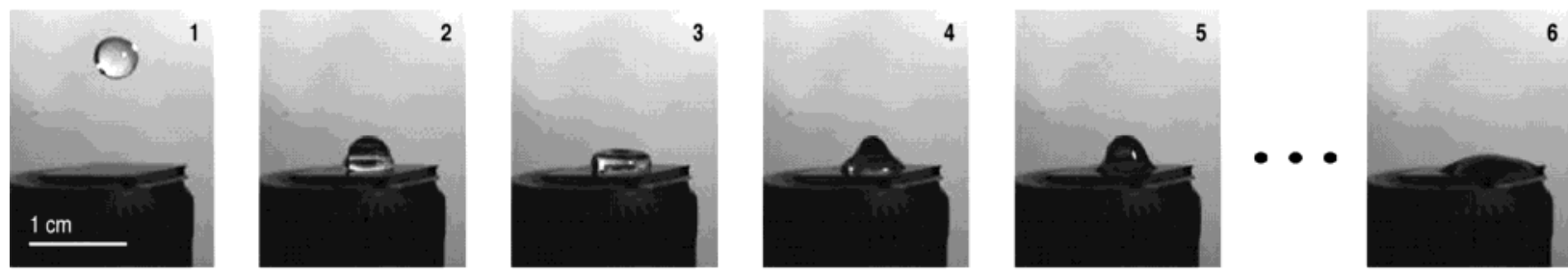

b
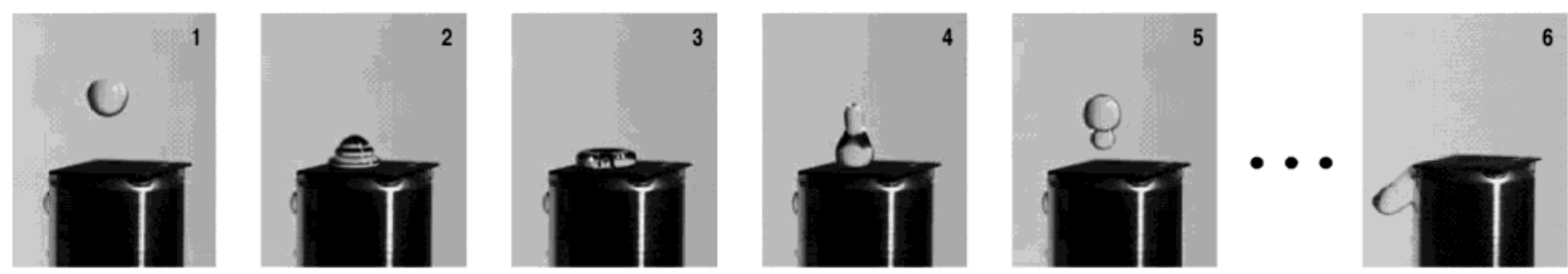

Figure 3. Time sequence images of a water droplet free falling on carbon nanotube forests. (a) As-grown forest in which the droplet eventually seeps through the forest, and (b) PTFE-coated forest in which the droplet eventually bounces off the forest. The droplets are approximately $3 \mathrm{~mm}$ in diameter, impacting the surfaces at a typical speed of $0.5 \mathrm{~m} / \mathrm{s}$. 
a

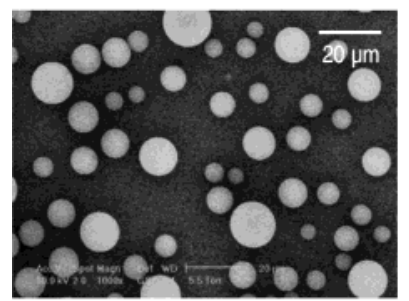

C

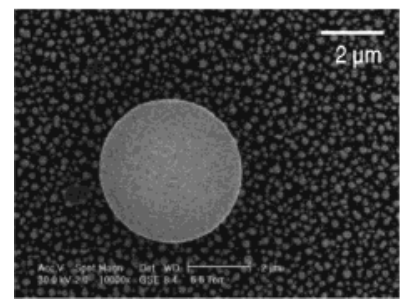

b

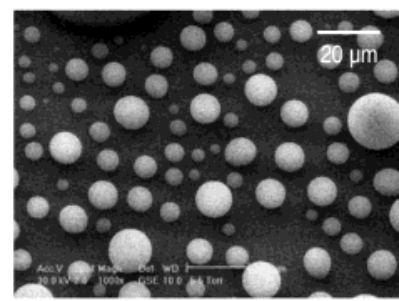

d

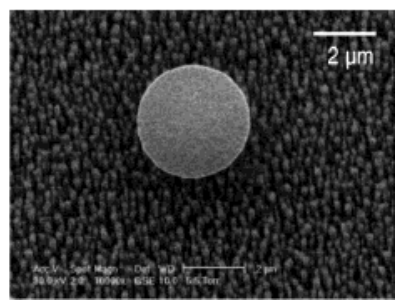

Figure 4. ESEM images of water droplets on carbon nanotube forests. (a) Top-down view of micron-sized water droplets suspended on the PTFE-coated forest, (b) $15^{\circ}$ tilt view at the same magnification, (c) top-down view of a single suspended water droplet in which the PTFE-coated nanotubes are also visible, and (d) $15^{\circ}$ tilt view at the same magnification.

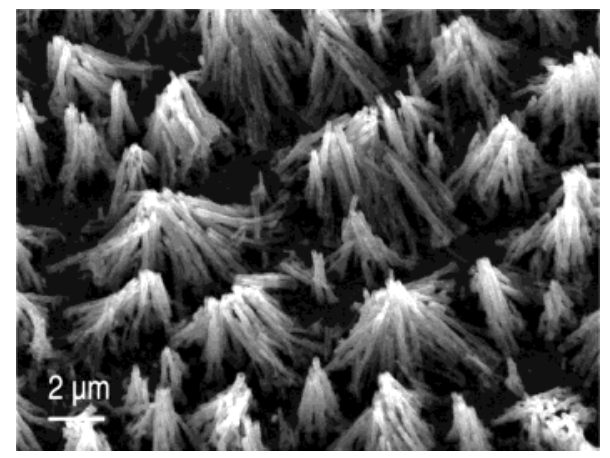

Figure 5. ESEM image of an as-grown forest without PTFE treatment, after exposure to water, showing the nanotubes bundling together as a result of the attractive capillary forces that arise during evaporative drying.

rough surface and the equilibrium contact angle $\theta$ obtained on a smooth surface of the same chemical composition:

$$
\cos \theta^{*}=-1+\phi_{\mathrm{s}}(\cos \theta+1)
$$

where the surface fraction $\phi_{\mathrm{s}}$ corresponds to the ratio of the surface of the top of the roughness in contact with the liquid with the apparent surface of the substrate. The value of $\theta$ relies on the Young's relation, $\cos \theta=\left(\gamma_{\mathrm{SV}}-\gamma_{\mathrm{SL}}\right) / \gamma_{\mathrm{LV}}$, where the $\gamma_{\mathrm{ij}}$ terms correspond to the solid-vapor, solidliquid, and liquid-vapor interfacial tensions, respectively. By analogy with porous wicking, eq 1 is expected to be valid for $\theta>90^{\circ}$, independent of the height of the rough structure. To probe the validity of the equation in our case, the value of the surface fraction $\phi_{\mathrm{s}}$ can be estimated from the pillar geometry of the forest:

$$
\phi_{\mathrm{s}}=n \pi r^{2}
$$

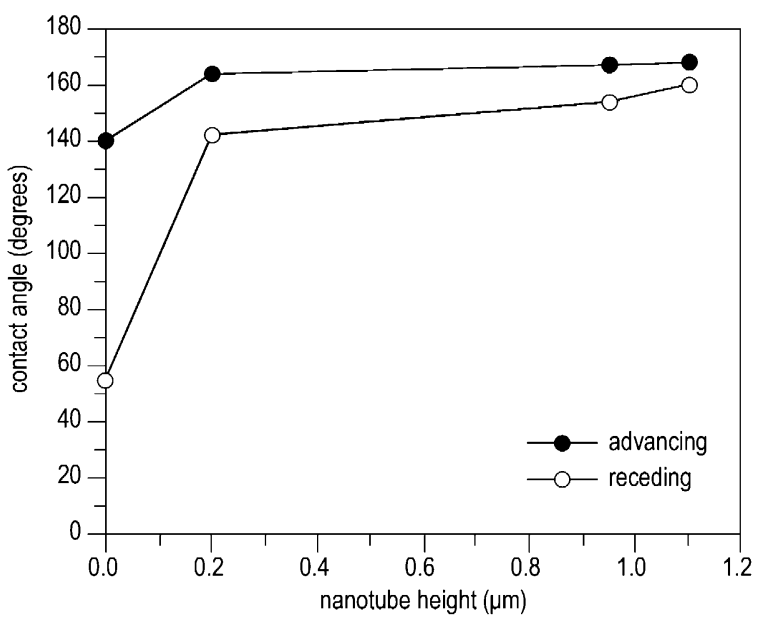

Figure 6. Dynamic water contact angle measurements on carbon nanotube forests. Hysteresis, the difference between the advancing and receding angles, decreases with increasing forest height, for the same nanotube diameter and spacing.

where $r$ is the radius of the tubes and $n$ the number of tubes per sample area. In the example illustrated in Figure 4, the tubes have an average radius of $60 \mathrm{~nm}$ (coated) and a density of 10 nanotubes per $\mu \mathrm{m}^{2}$, which yields a value for $\phi_{\mathrm{s}}$ of $11 \%$ from eq 2 . The advancing and receding contact angles of water on a smooth silicon wafer coated with PTFE are $150^{\circ}$ and $110^{\circ}$, respectively. These higher values compared to conventional, smooth PTFE indicate some inherent rough texture in the PTFE made using the HFCVD process. ${ }^{21}$ Using these values, the advancing and receding contact angles on the coated forest as predicted from eq 1 are therefore $170^{\circ}$ and $158^{\circ}$, respectively. This is in good agreement with the experimentally measured values of $170^{\circ}$ and $160^{\circ}$, respectively.

Finally, we evaluated the superhydrophobicity of a series of even shorter and narrower nanotube forests in dynamic mode, measuring the advancing and receding angles and hysteresis as a more sensitive way to assess wettability. Figure 6 shows the results for forests of nanotubes $50 \mathrm{~nm}$ in diameter (coated) with heights ranging from 0.2 to $1.1 \mu \mathrm{m}$, as well as for a plain substrate with the nickel catalyst but without any nanotube growth. We observe that the advancing angles are significantly higher for the forests than for the plain substrate, even at the shortest height, and reaches a value of $168^{\circ}$ at the tallest. However, the receding angles are more sensitive to differences in nanotube height, with hysteresis (difference between the advancing and receding angles) becoming smaller as the height of the forest increases, the tallest forest showing a hysteresis of $8^{\circ}$. This decrease in hysteresis may be a result of a decrease in interaction of the water droplet with the base surface. There seems to be a strong attraction of the water to the base surface since a strong hysteresis is observed just for the plain substrate without nanotubes. The water seepage into the uncoated nanotube forest also supports this view. By coating the nanotube forests with PTFE, we are able to introduce superhydrophobicity to forests with much shorter heights (down to $0.2 \mu \mathrm{m}$ ) than is possible without the PTFE treatment. This superhydrophobicity is also more stable, 
showing no signs of water seepage even after prolonged periods of time.

We have successfully created superhydrophobic carbon nanotube forests by modifying the surface of vertically aligned nanotubes with a PTFE coating. From our results, it is apparent that both the surface roughness templated by the nanotube forest and the low surface energy imparted by the PTFE coating are necessary components to achieve a stable superhydrophobic surface. The ability to use our HFCVD process to modify the surfaces of nanotubes directly is certainly attractive. It is unclear at this point how the PTFE polymer chains are attached to the nanotube surface. There is evidence that carbene radicals are sufficiently reactive to add directly to the $\mathrm{sp}^{2}$-hybridized carbons, ${ }^{28,29}$ so it is conceivable that the difluorocarbene radicals may attach covalently to the nanotube surface and subsequently polymerize from these sites. Regardless, HFCVD on a broader scope may be an important way to functionalize the surfaces of carbon nanotubes. Besides PTFE, HFCVD is able to make other common polymers including organosilicones ${ }^{30,31}$ and fluorosilicones. ${ }^{32,33}$ These and other functionalities may be useful for dispersing and separating carbon nanotubes since carbon nanotubes are notorious for their insolubility and tendency to aggregate into bundles. HFCVD would be helpful in many applications, including fillers for nanocomposites and single strand conductors in molecular electronics, where a need for separability is highly desirable.

Acknowledgment. This work was supported by the Cambridge-MIT Institute (CMI) Project of Carbon Nanotube Enabled Materials. K.B.K.T. also acknowledges the support of Christ's College, Cambridge. This work in part made use of MRSEC Shared Facilities at MIT supported by the National Science Foundation under Award Number DMR9400334.

\section{References}

(1) Iijima, S. Nature 1991, 354, 56-58

(2) Wong, E. W.; Sheehan, P. E.; Lieber, C. M. Science 1997, 277, 1971-1975.

(3) Tans, S. J.; Devoret, M. H.; Dal, H.; Thess, A.; Smalley, R. E.; Geerligs, L. J.; Dekker, C. Nature 1997, 386, 474-477.

(4) Hone, J.; Batlogg, B.; Benes, Z.; Johnson, A. T.; Fischer, J. E. Science 2000, 289, 1730-1734.
(5) Baughman, R. H.; Zakhidov, A. A.; de Heer, W. A. Science 2002, 297, 787-792

(6) Barthlott, W.; Neinhuis, C. Planta 1997, 202, 1-8.

(7) Blossey, R. Nature Mater. 2003, 2, 301-306.

(8) Feng, L.; Li, S.; Li, Y.; Li, H.; Zhang, L.; Zhai, J.; Song, Y.; Liu, B.; Jiang, L.; Zhu, D. Adv. Mater. 2002, 14, 1857-1860.

(9) Bico, J.; Marzolin, C.; Quéré, D. Europhys. Lett. 1999, 47, 220226.

(10) Lafuma, A.; Quéré, D. Nature Mater. 2003, 2, 457-460.

(11) Quéré, D. Nature Mater. 2002, 1, 14-15.

(12) Fox, H. W.; Zisman, W. A. J. Colloid Sci. 1950, 5, 514-531.

(13) Ren, Z. F.; Huang, Z. P.; Xu, J. W.; Wang, J. H.; Bush, P.; Siegel, M. P.; Provencio, P. N. Science 1998, 282, 1105-1107.

(14) Teo, K. B. K.; Chhowalla, M.; Amaratunga, G. A. J.; Milne, W. I.; Hasko, D. G.; Pirio, G.; Legagneux, P.; Wyczisk, F.; Pribat, D. Appl. Phys. Lett. 2001, 79, 1534-1536.

(15) Chhowalla, M.; Teo, K. B. K.; Ducati, C.; Rupesinghe, N. L.; Amaratunga, G. A. J.; Ferrari, A. C.; Roy, D.; Robertson, J.; Milne, W. I. J. Appl. Phys. 2001, 90, 5308-5317.

(16) Fowkes, F. M.; Harkins, W. D. J. Am. Chem. Soc. 1940, 62, 33773386.

(17) Morcos, I. J. Chem. Phys. 1972, 57, 1801-1802.

(18) Li, H.; Wang, X.; Song, Y.; Liu, Y.; Li, Q.; Jiang, L.; Zhu, D. Angew. Chem., Int. Ed. 2001, 40, 1743-1746.

(19) Li, S.; Li, H.; Wang, X.; Song, Y.; Liu, Y.; Jiang, L.; Zhu, D. J. Phys. Chem. B 2002, 106, 9274-9276.

(20) Limb, S. J.; Labelle, C. B.; Gleason, K. K.; Edell, D. J.; Gleason, E. F. Appl. Phys. Lett. 1996, 68, 2810-2812.

(21) Lau, K. K. S.; Caulfield, J. A.; Gleason, K. K. Chem. Mater. 2000, 12, 3032-3037.

(22) Liang, C. Y.; Krimm, S. J. Chem. Phys. 1956, 25, 563-571.

(23) Moynihan, R. E. J. Am. Chem. Soc. 1959, 81, 1045-1050.

(24) Videos of dynamic water experiments on uncoated and PTFE-coated carbon nanotube forests can be viewed from the web page link at http://web.mit.edu/gleasongroup/cnt/cntwater.html.

(25) Richard, D.; Quéré, D. Europhys. Lett. 2000, 50, 769-775.

(26) Richard, D.; Clanet, C.; Quéré, D. Nature 2002, 417, 811.

(27) Cassie, A. B. D.; Baxter, S. Trans. Faraday Soc. 1944, 40, 546551.

(28) Chen, J.; Hamon, M. A.; Hu, H.; Chen, Y.; Rao, A. M.; Eklund, P. C.; Haddon, R. C. Science 1998, 282, 95-98.

(29) Chen, Y.; Haddon, R. C.; Fang, S.; Rao, A. M.; Eklund, P. C.; Lee, W. H.; Dickey, E. C.; Grulke, E. A.; Pendergrass, J. C.; Chavan, A.; Haley, B. E.; Smalley, R. E. J. Mater. Res. 1998, 13, 2423-2431.

(30) Lau, K. K. S.; Pryce Lewis, H. G.; Limb, S. J.; Kwan, M. C.; Gleason, K. K. Thin Solid Films 2001, 395, 288-291.

(31) Lewis, H. G. P.; Casserly, T. B.; Gleason, K. K. J. Electrochem. Soc. 2001, 148, F212-F220.

(32) Murthy, S. K.; Gleason, K. K. Macromolecules 2002, 35, 19671972.

(33) Murthy, S. K.; Olsen, B. D.; Gleason, K. K. Langmuir 2002, 18, 6424-6428.

NL034704T 